\title{
Intensive glucose lowering and the risk of vascular events and premature death in patients with decreased kidney function: the ADVANCE trial
}

\section{Authors:}

Toshiaki Ohkuma ${ }^{1}$, Sophia Zoungas ${ }^{1,2}$, Min Jun ${ }^{1}$, Liu Lisheng ${ }^{3}$, Giuseppe Mancia ${ }^{4}$, Michel Marre $^{5}$, Anthony Rodgers ${ }^{1}$, Bryan Williams ${ }^{6}$, Mark Woodward ${ }^{1,7,8}$, John Chalmers ${ }^{1}$, on behalf of the ADVANCE Collaborative Group

\section{Affiliations:}

1. The George Institute for Global Health, University of New South Wales, Sydney, Australia

2. School of Public Health and Preventive Medicine, Monash University, Melbourne,

Australia

3. The Chinese Hypertension League Institute, Beijing, China

4. Istituto Auxologico Italiano, University of Milan-Bicocca, Italy

5. Department of Endocrinology, Hopital Bichat-Claude Bernard, Universite Paris, France

6. Institute of Cardiovascular Sciences, University College London and National Institute of Health Research UCL Hospitals Biomedical Research Centre, London, UK

7. The George Institute for Global Health, University of Oxford, Oxford, UK

8. Department of Epidemiology, Johns Hopkins University, Baltimore MD, USA

This article has been accepted for publication and undergone full peer review but has not been through the copyediting, typesetting, pagination and proofreading process which may lead to differences between this version and the Version of Record. Please cite this article as doi: $10.1111 /$ dom. 13878 


\section{Corresponding author:}

John Chalmers

The George Institute for Global Health, University of New South Wales, Sydney, Australia

Level 10, King George V Building, Royal Prince Alfred Hospital, Missenden Rd,

Camperdown NSW 2050 Australia

Email: chalmers@georgeinstitute.org.au

Phone: +61 280524586 


\begin{abstract}
To assess effects of intensive glucose-control on the risk of major clinical outcomes according to estimated glomerular filtration rate (eGFR) levels in type 2 diabetes. Among 11,140 ADVANCE trial participants, 11,096 participants with baseline eGFR measurements were included, classified into three groups: eGFR $\geq 90,60-89$, and $<60 \mathrm{ml} / \mathrm{min} / 1.73 \mathrm{~m}^{2}$. Relative risk reduction of randomised intensive glucose-control on the composite of major macro- and microvascular events, all-cause death, and cardiovascular death did not significantly vary by eGFR levels ( $\mathrm{p}$ for heterogeneity $\geq 0.49$ ). The risk of severe hypoglycaemia increased with intensive glucose-control; however, this risk did not vary across eGFR groups ( $\mathrm{p}$ for heterogeneity=0.83). The risk-benefit profile of intensive glucosecontrol in patients with type 2 diabetes and impaired kidney function appears comparable to that observed in those with preserved kidney function.
\end{abstract}

Clinical trial registration number: NCT00145925, ClinicalTrials.gov 


\section{Introduction}

Glycaemic control reduces the risk of long-term diabetic complications and thus has a central role in diabetes care. ${ }^{1}$ However, there is ongoing uncertainty as to the risk-benefit balance of intensive glucose lowering, particularly with respect to premature death in patients with chronic kidney disease (CKD)., ${ }^{2,3}$ Although, dose adjustment of glucose lowering therapies occurs in the setting of decline in kidney function, the effects of intensive glucose lowering on clinical outcomes across different levels of kidney function remain unclear.

The aim of this study was to examine whether the effects of intensive glucose lowering differ across different level of kidney function defined by eGFR in type 2 diabetes.

\section{Methods}

Study design and population

The Action in Diabetes and Vascular Disease: Preterax and Diamicron Modified Release Controlled Evaluation (ADVANCE) trial was a factorial randomised controlled trial to evaluate the effects of intensive blood glucose lowering treatment and blood pressure lowering on vascular outcomes in patients with type 2 diabetes (ClinicalTrials.gov number, NCT00145925). ${ }^{4-6}$ Briefly, 11,140 individuals with type 2 diabetes at high risk of cardiovascular events were enrolled from 215 centres in 20 countries, and were randomised to either a gliclazide (modified release)-based intensive glucose-control (target HbA1c $\leq 6.5 \%)$ or standard glucose-control based on local guideline of participating countries and to either a fixed-dose combination of perindopril $(2 \mathrm{mg})$ and indapamide $(0.625 \mathrm{mg})$ or matching placebo after a 6-week run-in period, during which they continued their usual 
methods of glucose-control and received perindopril and indapamide in a fixed combination. Dosing and titration of glucose-control therapy during the trial was at the discretion of the responsible physician. Participants were followed up for a median of 5.0 years. Ethics approval for the trial was obtained from each centre's institutional review board. All participants provided written informed consent.

eGFR evaluations

eGFR was calculated by the CKD-EPI equation. ${ }^{7}$ Only participants with baseline eGFR measurements were included $(n=11,096)$, and were classified into 3 groups: eGFR $\geq 90$, $60-<90,<60 \mathrm{ml} / \mathrm{min} / 1.73 \mathrm{~m}^{2}$ ), guided by international staging along of $\mathrm{CKD},{ }^{8}$ whilst keeping analysis groups reasonably large.

\section{Outcomes}

The outcomes assessed in this analysis were 1) the composite of major macrovascular (death from cardiovascular causes, nonfatal myocardial infarction, or nonfatal stroke) and major microvascular (new or worsening nephropathy or retinopathy) events, 2) all-cause death, 3) cardiovascular death, 4) major coronary events, 5) major cerebrovascular events, 6) new or worsening nephropathy, 7) new or worsening retinopathy, and 8) severe hypoglycaemia, as previously defined. ${ }^{6}$

\section{Statistical analysis}


Linear trends of baseline characteristics across categories were tested by linear regression analysis and logistic regression analysis, as appropriate. Mean $\mathrm{Hb}_{1 \mathrm{c}}$ levels during the follow-up period were calculated by linear mixed models, according to randomised treatment and baseline eGFR levels. The effects of randomised treatment on outcomes were assessed by unadjusted Cox regression models according to subgroups defined by baseline eGFR, based on the intension-to-treat principle. Heterogeneity in treatment effects across subgroups was tested by adding interaction terms to the relevant models. Sensitivity analyses were conducted, in which subgroups were defined as eGFR of $\geq 90,60-<90,45-<60$, and $<45$ $\mathrm{ml} / \mathrm{min} / 1.73 \mathrm{~m}^{2}$. We also performed additional analyses, in which participants were grouped according to urine albumin-to-creatinine ratio (UACR, $<30,30-150, \geq 150 \mu \mathrm{g} / \mathrm{mg}$ ) or The Kidney Disease: Improving Global Outcomes (KDIGO) risk categories ${ }^{8}$ based on both eGFR and UACR (low, moderate, high/very high). Statistical analyses were performed with SAS 7.11 (SAS Institute, Cary NC, USA). A two-sided p-value $<0.05$ was considered statistically significant.

\section{Results}

Baseline characteristics

Of 11,096 participants, $22 \%$ had an eGFR $\geq 90 \mathrm{ml} / \mathrm{min} / 1.73 \mathrm{~m}^{2}, 56 \%$ had an eGFR 60 $<90 \mathrm{ml} / \mathrm{min} / 1.73 \mathrm{~m}^{2}$, and $22 \%$ had an eGFR $<60 \mathrm{ml} / \mathrm{min} / 1.73 \mathrm{~m}^{2}$ at baseline (Supplementary Table 1). Participants with lower eGFR were more likely to be older, have longer duration of diabetes, have a history of macro- and microvascular disease, have lower baseline $\mathrm{HbA}_{1 \mathrm{c}}$, higher BMI, and UACR levels, and less likely to be treated with metformin (all p for trend 
$<0.001)$. The proportion of patients treated with gliclazide, other sulfonylureas or insulin did not differ across subgroups ( $\mathrm{p}$ for trend $\geq 0.08$ ).

Randomised treatment effects of intensive glucose-control according to baseline eGFR

The mean $\mathrm{HbA}_{1 \mathrm{c}}$ levels during the follow-up period in intensive glucose-control were $6.70 \%, 6.66 \%$, and $6.71 \%$ in participants with eGFR $\geq 90,60-<90,<60 \mathrm{ml} / \mathrm{min} / 1.73 \mathrm{~m}^{2}$ (Figure 1). The corresponding values were higher in standard glucose control, with $7.42 \%$, $7.29 \%$, and $7.32 \%$ in each eGFR subgroup. Proportion of participants who were initiated insulin treatment during follow-up among those without insulin at baseline was lower in those with lower eGFR at baseline (Supplementary Table 2). When restricting to participants who have never treated with insulin throughout the trial period, similar reductions in $\mathrm{HbA}_{1 \mathrm{c}}$ levels were observed (Supplementary Figure 1).

Overall, intensive glucose-lowering significantly reduced the risk of the composite of major macrovascular and microvascular events (hazard ratio [HR] 0.89, 95\% CI 0.82-0.97, Figure 2) and there was no evidence of heterogeneity in the randomised effects across baseline eGFR levels (p for heterogeneity=0.51). The corresponding HRs (95\% CIs) for allcause death, cardiovascular death, major coronary events, major cerebrovascular events, new or worsening nephropathy, and new or worsening retinopathy were 0.93 (0.82-1.05), 0.87 (0.73-1.03), 0.91 (0.78-1.06), 0.96 (0.81-1.15), 0.78 (0.66, 0.93), and 0.95 (0.82, 1.10), with consistent risk reductions across eGFR subgroups (all $p$ for heterogeneity $\geq 0.21$ ), with the exception of new or worsening nephropathy ( $\mathrm{p}$ for heterogeneity $=0.03$ ). However, this heterogeneity was not evident when trend in the effects across subgroups was examined ( $p$ 
for trend=0.79). The risk of severe hypoglycaemia increased with intensive glucose-control (HR 1.86, 95\% CI 1.42-2.44) and this increased risk did not vary by baseline eGFR levels (p for heterogeneity=0.83). Results were broadly the same when participants with eGFR $<60$ $\mathrm{ml} / \mathrm{min} / 1.73 \mathrm{~m}^{2}$ were split into those with $<45$ and $45-<60 \mathrm{ml} / \mathrm{min} / 1.73 \mathrm{~m}^{2}$ (p for heterogeneity $\geq 0.13$, Supplementary Figure 2). Additional analyses, in which participants were classified by baseline UACR or KDIGO risk categories, also showed no significant heterogeneity in the effects of intensive glucose lowering across subgroups (Supplementary Figure 3 and 4).

\section{Conclusions}

In this analysis, intensive glucose lowering resulted in consistent treatment effects on vascular events and death among patients with type 2 diabetes across different levels of eGFR. Although the occurrence of severe hypoglycaemia was more common at lower eGFR levels, the risk associated with intensive glucose lowering was similar across eGFR subgroups. Our results suggest that the risk-benefit balance of intensive glucose-control in patients with type 2 diabetes with impaired kidney function may be comparable to that observed in patients with preserved kidney function.

The ACCORD trial previously reported that intensive glycaemic control in patients with type 2 diabetes with CKD significantly increased the risk of all-cause and cardiovascular mortality, compared with standard glycaemic control. ${ }^{2}$ One explanation for this may be an increased risk of severe hypoglycaemia in CKD. Severe hypoglycaemia is associated with an increased risk of death ${ }^{9,10}$ and cardiovascular events. ${ }^{9}$ Taken together, intensive glucose 
lowering in patients with CKD has been suggested to be detrimental and inappropriate. ${ }^{2}$ However, in the ACCORD trial, CKD subgroups were defined by both urinary albumin-tocreatinine ratio and $\mathrm{eGFR}^{2}$ and not solely by measures of kidney function which are directly associated with renal clearance and dosage adjustments of therapies in CKD. To our knowledge, the present study is the first to report on the consistency of the randomised treatment effects of intensive glucose lowering according to eGFR levels. Overall, intensive glucose-control reduced the risk of all-cause and cardiovascular death, as well as other vascular events, to a similar extent, regardless of baseline eGFR level. The relative risk of severe hypoglycaemia associated with intensive glucose lowering was also similar across eGFR subgroups. Similar tendencies were observed when participants were subgrouped based on baseline UACR or KDIGO risk categories (using both baseline eGFR and UACR), whereas the ACCORD trial showed evidence of harm associated with intensive glucoselowering in $\mathrm{CKD},{ }^{2}$ as mentioned above. The reason for the inconsistency is unclear, but it may be partly due to the difference in characteristics of study population and approaches to glucose-lowering. ${ }^{11,12}$ For example, participants in ACCORD have longer duration of diabetes (ACCORD: 10.9 years, ${ }^{2}$ ADVANCE: 7.9 years) and experienced severe hypoglycemia more frequently under intensive glucose-lowering ${ }^{11}$ (HR [95\% CIs], ACCORD: 3.07 [2.59, 3.63], ADVANCE: 1.86 [1.42, 2.44]), in comparison with those in ADVANCE. These findings suggest the long-term benefits of intensive glucose lowering may be observed even in patients with decreased kidney function, although careful attention to the occurrence of hypoglycaemia is required. Furthermore, more intensive glucose-control decreased $\mathrm{HbA}_{1 \mathrm{c}}$ levels even without insulin administration, thus demonstrating the 
potentially efficacy of glycaemic intensification without initiation of insulin in patients with decreased kidney function. This may have important clinical implications for preventing vascular events and premature death in these patients.

The strengths of this study include the large sample recruited internationally, longterm follow-up, and rigorous central adjudication of the outcomes, which enabled precise assessment of the randomised treatment effects at different eGFR levels. However, study participants consisted of those enrolled in a randomised trial, which may limit the applicability of the results to broader general populations with diabetes.

In conclusion, our results suggest that an intensive glycaemic control strategy may be recommended for patients with type 2 diabetes with impaired kidney function as well as those with preserved kidney function. Attention to prevention of hypoglycaemia is warranted across all levels of kidney function. 


\section{Acknowledgements}

\section{Funding}

The ADVANCE trial was funded by the grants from the National Health and Medical Research Council (NHMRC) of Australia and Servier. TO is supported by the John Chalmers Clinical Research Fellowship of the George Institute. MJ is supported by a Scientia Fellowship from UNSW Sydney. BW is a National Institute for Health Research (NIHR) Senior Investigator. MW is a NHMRC of Australia Principal Research Fellow (1080206).

\section{Conflict of Interest}

TO and LL report no conflicts of interest. SZ reports participation in advisory boards, expert committees or educational meetings outside the submitted work on behalf of Monash University for Boehringer-Ingelheim, Sanofi, AstraZeneca, Novo Nordisk, and MSD Australia (payment to institution). MJ reports receiving grant support from the National Health and Medical Research Council of Australia (Project Grant: 1148060) and unrestricted grant support from VentureWise (a wholly owned commercial subsidiary of NPS MedicineWise) to conduct a commissioned project funded by AstraZeneca. GM reports personal fees from Servier Laboratories, Bayer, Boehringer Ingelheim, Daiichi Sankyo, Medtronic, Novartis, Menarini Group, Recordati, and Takeda Pharmaceutical Company. MM received personal fees from Novo Nordisk, Sanofi, Eli Lilly, Merck Sharp and Dohme, Abbott, Novartis, Servier, and AstraZeneca and grant support from Novo Nordisk, Sanofi, Eli Lilly, Merck Sharp and Dohme and Novartis. AR receives salary in part from George Health Enterprises, the social enterprise arm of The George Institute. George Health Enterprises has 
received investment to develop fixed-dose combinations containing aspirin, statin, and blood pressure lowering drugs. BW reports speaker fees for Servier, Novartis, Daiichi Sankyo, Pfizer and Boehringer Ingelheim and serves on trial steering committees for Novartis, Relypsa and Vascular Dynamics. MW reports consultancy fees from Amgen and Kirin and is supported by an Australian National Health and Medical Research Council fellowship (APP1080206) and Program Grant (APP1149987). JC received research grants from the

National Health and Medical Research Council of Australia and from Servier for the ADVANCE trial and ADVANCE-ON post-trial follow-up, and honoraria for speaking about these studies at scientific meetings, and reports grant support from Program Grant APP1149987 from the National Health and Medical Research Council of Australia.

\section{Author Contributions}

TO, SZ, MJ, MW and JC contributed to the concept and rationale for the study and interpretation of the results, and drafted the manuscript. TO conducted statistical analysis with advice from MW. All authors contributed to discussion and reviewed and edited the manuscript. JC is the guarantor of this work and, as such, had full access to all the data in the study and takes responsibility for the integrity of the data and the accuracy of the data analysis. 


\section{References}

1. Davies MJ, D'Alessio DA, Fradkin J, et al. Management of Hyperglycemia in Type 2 Diabetes, 2018. A Consensus Report by the American Diabetes Association (ADA) and the European Association for the Study of Diabetes (EASD). Diabetes Care. 2018;41(12):2669-2701.

2. Papademetriou V, Lovato L, Doumas M, et al. Chronic kidney disease and intensive glycemic control increase cardiovascular risk in patients with type 2 diabetes. Kidney Int. 2015;87(3):649-659.

3. Wong MG, Perkovic V, Chalmers J, et al. Long-term Benefits of Intensive Glucose Control for Preventing End-Stage Kidney Disease: ADVANCE-ON. Diabetes Care. 2016;39(5):694-700.

4. ADVANCE Management Committee. Study rationale and design of ADVANCE: action in diabetes and vascular disease--preterax and diamicron MR controlled evaluation. Diabetologia. 2001;44(9):1118-1120.

5. Patel A, MacMahon S, Chalmers J, et al. Effects of a fixed combination of perindopril and indapamide on macrovascular and microvascular outcomes in patients with type 2 diabetes mellitus (the ADVANCE trial): a randomised controlled trial. Lancet. 2007;370(9590):829-840. 
6. Patel A, MacMahon S, Chalmers J, et al. Intensive blood glucose control and vascular outcomes in patients with type 2 diabetes. $N$ Engl $J$ Med. 2008;358(24):2560-2572.

7. Levey AS, Stevens LA, Schmid CH, et al. A new equation to estimate glomerular filtration rate. Ann Intern Med. 2009;150(9):604-612.

8. Kidney Disease: Improving Global Outcomes (KDIGO) CKD Work Group. KDIGO 2012 Clinical practice guideline for the evaluation and management of chronic kidney disease. Kidney Int Suppl. 2013;3(1):1-150.

9. Zoungas S, Patel A, Chalmers J, et al. Severe hypoglycemia and risks of vascular events and death. $N$ Engl J Med. 2010;363(15):1410-1418.

10. Bonds DE, Miller ME, Bergenstal RM, et al. The association between symptomatic, severe hypoglycaemia and mortality in type 2 diabetes: retrospective epidemiological analysis of the ACCORD study. BMJ. 2010;340:b4909.

11. Turnbull FM, Abraira C, Anderson RJ, et al. Intensive glucose control and macrovascular outcomes in type 2 diabetes. Diabetologia. 2009;52(11):2288-2298.

12. Standards of Medical Care in Diabetes-2019. Diabetes Care. 2019;42(Suppl 1):S1186. 


\section{Figure legends}

Figure 1. Mean $\mathrm{HbA}_{1 \mathrm{c}}$ levels at baseline and during follow-up according to glucosecontrol strategy and baseline eGFR.

Mean values during follow-up were presented as mean (SE).

Figure 2. Randomised effects of intensive glucose control on the risk of major clinical outcomes according to baseline eGFR.

White diamonds indicate the HRs for subgroups defined by baseline eGFR.

Black diamonds indicate the HRs of overall for the current study.

Abbreviations: CI, confidence interval; eGFR, estimated glomerular filtration rate. 


\section{Figure 1}

A) eGFR $\geq 90\left(\mathrm{ml} / \mathrm{min} / 1.73 \mathrm{~m}^{2}\right)$

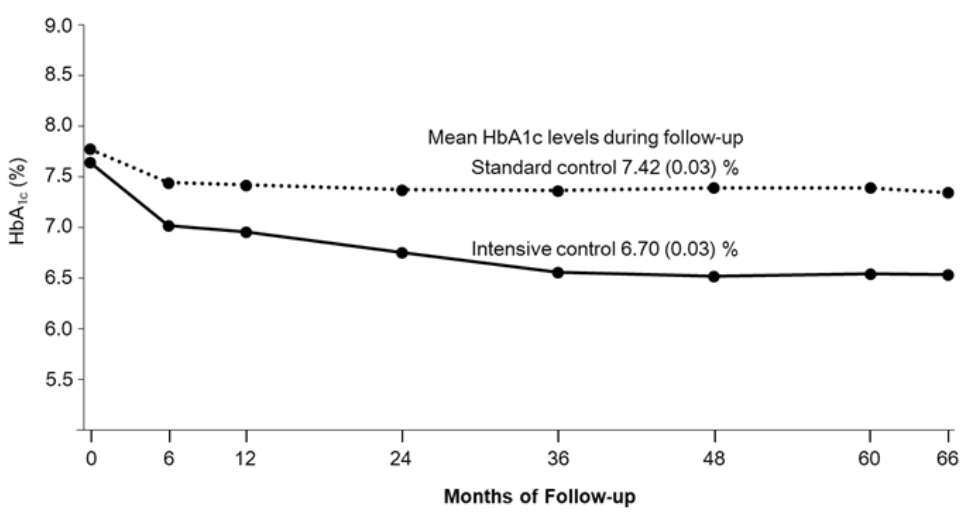

B) eGFR $60-<90\left(\mathrm{ml} / \mathrm{min} / 1.73 \mathrm{~m}^{2}\right)$

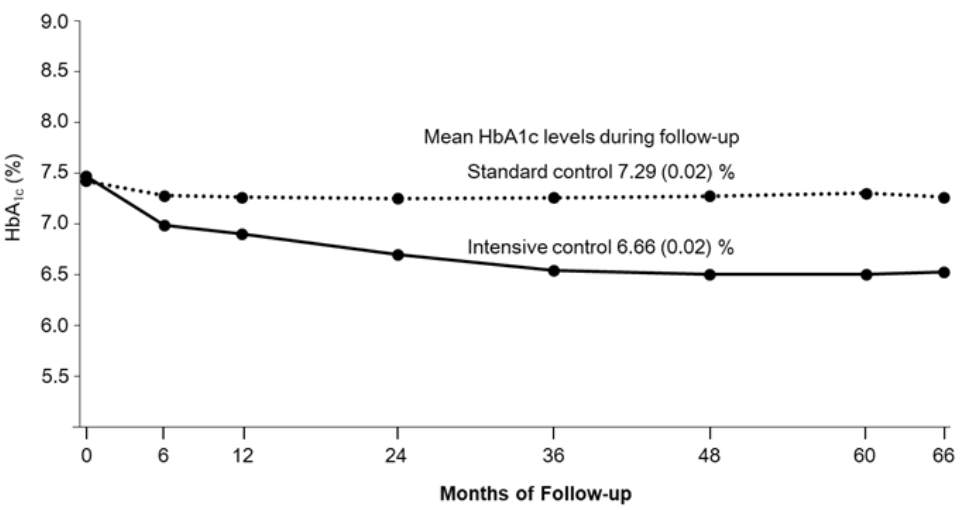

C) eGFR $<60\left(\mathrm{ml} / \mathrm{min} / 1.73 \mathrm{~m}^{2}\right)$

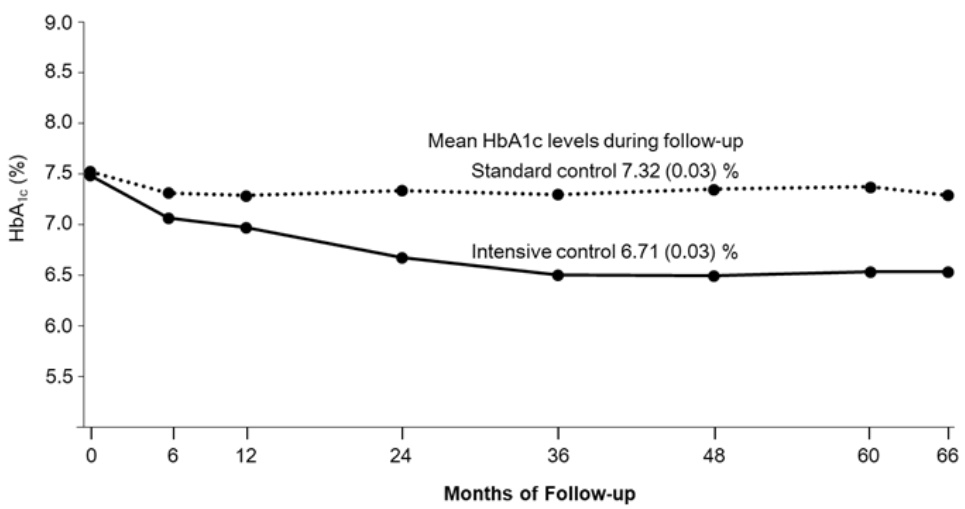


Figure 2

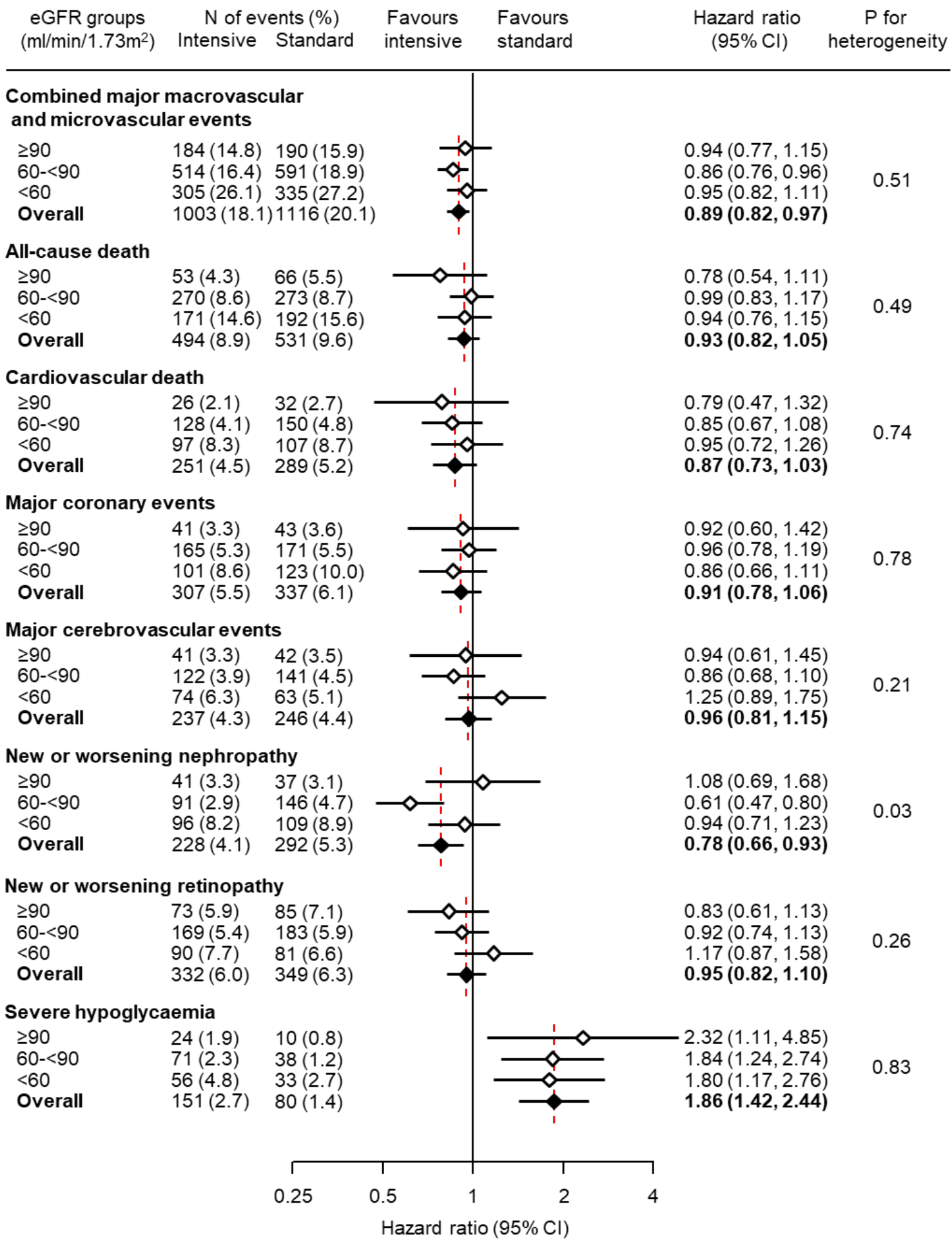

